\title{
Analysis of Inner Ear Anomalies in Unilateral Congenital Aural Atresia Combined With Microtia
}

\author{
Euyhyun Park (i) $\cdot$ Gijeong Lee $\cdot$ Hak Hyun Jung $\cdot$ Gi Jung Im (i) \\ Department of Otolaryngology-Head and Neck Surgery, Korea University College of Medicine, Seoul, Korea
}

Objectives. The aim of this study was to analyze the incidence of inner ear anomalies in patients with unilateral congenital aural atresia (CAA) combined with microtia.

Methods. We retrospectively reviewed 61 patients with unilateral CAA combined with microtia who underwent high-resolution temporal bone computed tomography (TBCT) and hearing examination. Inner ear anomalies were analyzed using TBCT and evaluated according to the Jahrsdoerfer grading system, Marx classification, and extent of inferior displacement of the mastoid tegmen.

Results. Inner ear anomalies were observed in 14 patients (23.0\%). Lateral semicircular canal (LSCC) dysplasia was the most common inner ear anomaly, with an incidence of $16.4 \%$. The incidence was significantly higher on the pathologic side than on the unaffected side $(P=0.002)$. All vascular anomalies were observed in the high-riding jugular bulb, with an incidence of $24.6 \%$. The incidence was significantly higher on the pathologic side than on the unaffected side $(P<0.001)$. LSCC dysplasia was significantly more common in patients with a lower Jahrsdoerfer score (odds ratio, $0.66 ; P=0.004)$.

Conclusion. The incidence of inner ear anomalies was relatively high in patients with unilateral CAA combined with microtia; LSCC dysplasia was the most common anomaly and the probability of coexistence was higher in patients with a lower Jahrsdoerfer score.

Keywords. Inner Ear; Anomalies; Aural Atresia, Congenital; Congenital Microtia; Semicircular Canals

\section{INTRODUCTION}

Congenital aural atresia (CAA) is defined as a congenital malformation of the external auditory canal (EAC) and is often associated with anomalous features of the external, middle, and inner ear structures [1]. Microtia is a congenital malformation of the external ear resulting in an auricular deformity and is typically observed with CAA [2]. CAA and microtia, which are relatively common in craniofacial microsomia, arise from developmental disorders involving the first and second branchial arches [3]. The prevalence of CAA is estimated to be one per 3,300 to

- Received June 5, 2018

Revised September 1, 2018

Accepted September 14, 2018

- Corresponding author: Gi Jung Im

Department of Otolaryngology-Head and Neck Surgery, Korea University

College of Medicine, 73 Inchon-ro, Seongbuk-gu, Seoul 02841, Korea

Tel: +82-2-920-5486, Fax: +82-2-925-5233

E-mail: logopas@korea.ac.kr
10,000 , with the unilateral type reported to be three to five times more common than the bilateral type [4,5]. The incidence of inner ear anomalies reported in patients with CAA varies between $3 \%$ and $22 \%$, and differs with higher incidences occurring with the presence of comorbidities and bilateral malformations [3,6-8]. Vestibular dysplasia (particularly in the semicircular canal) is estimated to be the most common inner ear anomaly in CAA patients [3].

Most patients with CAA combined with microtia exhibit conductive hearing loss because of a stenotic EAC and ossicular anomalies [9]. Therefore, atresiaplasty and active middle ear implants (aMEI) are current treatment options for hearing rehabilitation in patients with CAA [10]. Patient selection is critical when deciding on the treatment modality for CAA. The use of temporal bone computed tomography (TBCT) analysis is generally based on the Jahrsdoerfer grading system [11]. Although this grading system is widely used, other factors have been considered, such as the size of the incudostapedial (IS) joint angle

Copyright (C) 2019 by Korean Society of Otorhinolaryngology-Head and Neck Surgery.

This is an open-access article distributed under the terms of the Creative Commons Attribution Non-Commercial License (http://creativecommons.org/licenses/by-nc/4.0)

which permits unrestricted non-commercial use, distribution, and reproduction in any medium, provided the original work is properly cited. 
and the extent of inferior displacement of the mastoid tegmen $[12,13]$. Patients with narrow IS joint angles $\left(<120^{\circ}\right)$ have been demonstrated to have more favorable hearing outcomes compared with patients with broad angles $\left(>120^{\circ}\right)$, and the extent of inferior displacement of the mastoid tegmen is related to the severity of CAA [14]. Recent studies have attempted to document the clinical factors associated with CAA.

To our knowledge, few reports have analyzed inner ear anomalies that are limited to patients with unilateral CAA combined with microtia. Therefore, we report the incidence of inner ear anomalies, detected using high-resolution TBCT, based on a comparison of the pathologic and unaffected sides. Furthermore, we evaluated the clinical manifestations associated with inner ear anomalies in patients with unilateral CAA combined with microtia.

\section{MATERIALS AND METHODS}

This was a retrospective review of patients with unilateral CAA combined with microtia from 2004 to 2015 at a tertiary-level referral hospital. Medical records, including results from highresolution TBCT, were reviewed. This study was approved by the Institutional Review Board of Korea University Anam Hospital (IRB No. AN09162-2), and a written informed consent was obtained from all participants.

Patients who met the following inclusion criteria were included: (1) presence of unilateral CAA combined with microtia, (2) underwent high-resolution TBCT, and (3) underwent pure tone audiometry (PTA). Cases of craniofacial malformations other than unilateral CAA combined with microtia were excluded from the study. TBCT was performed by a trained physician using a multidetector computed tomography device capable of capturing high-resolution images (64-detector row scanner; tube voltage, $140 \mathrm{kV}$; tube current, $300 \mathrm{mAs}$; field of view, $205 \mathrm{~mm}$; slice thickness, $0.7 \mathrm{~mm}$; slice interval, $0.7 \mathrm{~mm}$; reconstruction algorithms, ultra-sharp (bony) algorithm; Somatom definition flash, Siemens, Erlangen, Germany). An experienced otologist and radiologist (GJI, SHY) jointly reviewed the TBCT images.

\section{H}

- The incidence of inner ear anomalies was relatively high $(23.0 \%)$ in patients with unilateral congenital aural atresia (CAA) combined with microtia.

- Lateral semicircular canal (LSCC) dysplasia was the most common $(16.4 \%)$ anomaly in patients with unilateral CAA combined with microtia.

- The Jahrsdoerfer score would show a relationship with the presence of LSCC dysplasia in patients with unilateral CAA combined with microtia. (odds ratio, $0.66 ; P=0.004$ ).

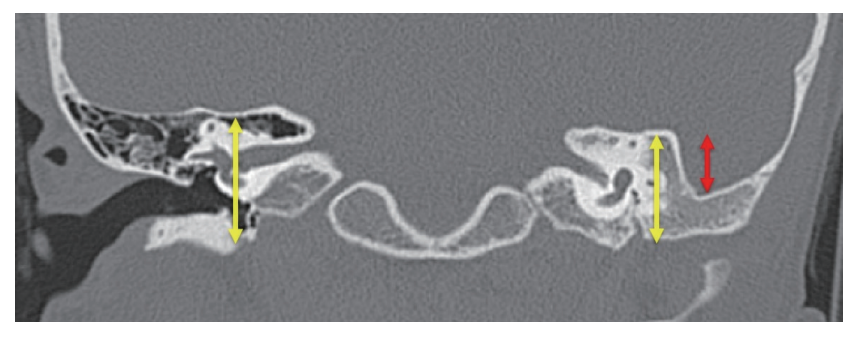

Fig. 1. The extent of inferior displacement of the mastoid tegmen was calculated as the ratio of middle ear height to mastoid tegmen displacement (yellow arrows, middle ear height; red arrow, mastoid tegmen displacement).

The Jahrsdoerfer score, Marx grade, and extent of inferior displacement of the mastoid tegmen were determined. The Jahrsdoerfer score was determined based on (1) stapes being present, (2) oval window being open, (3) round window being present and open, (4) middle ear space being large and favorable, (5) malleus-incus complex being present, (6) mastoid being wellpneumatized, (7) incus and stapes being connected, (8) facial nerve position, and (9) appearance of external ear being normal [11]. The external ear was evaluated according to the Marx classification: abnormal auricle with all identifiable landmarks classified as grade 1 , abnormal auricle without identifiable landmarks classified as grade 2 , and very small auricular tags or anotia classified as grade 3 [2]. The extent of inferior displacement of the mastoid tegmen was calculated from the middle ear height, measured as the vertical distance between the roof of the epitympanum and the floor of the hypotympanum. The length from the same height as the roof of the epitympanum to the mastoid tegmen was measured and the ratio of this to the middle ear height was calculated (Fig. 1) [14].

The following inner ear structures and anomalies were analyzed according to the classification proposed by Sennaroglu and Saatci [15]: (1) cochlear dysplasia (Michel deformity, cochlear aplasia, common cavity deformity, cochlear hypoplasia, incomplete partition type I, and incomplete partition type II), (2) vestibular dysplasia (Michel deformity, common cavity, absent vestibule, hypoplastic vestibule, and dilated vestibule), (3) semicircular canal dysplasia (absent, hypoplastic, or enlarged), (4) internal auditory canal (IAC) dysplasia (absent, narrow, or enlarged), and (5) vestibular and cochlear aqueduct findings (enlarged or normal). Facial nerve anomalies and the presence of a high-riding jugular bulb (HRJB) were also evaluated. A jugular bulb that extended into the middle ear, above the level of the floor of the IAC or tympanic annulus, was defined as a HRJB [16].

Data are presented as the mean \pm standard deviation. The demographic data were compared between the pathologic side and the unaffected side by using the paired $t$-test, and inner ear anomalies were compared between the pathologic side and the unaffected side by using the chi-square test (or Fisher exact test as appropriate). Variables found to be statistically significant ac- 
cording to univariate analysis were then entered into multiple logistic regression analysis to identify the clinical manifestations in patients with unilateral CAA combined with microtia. A probability value of $<0.05$ was considered statistically significant. All data were analyzed using the IBM SPSS ver. 22.0 (IBM SPSS Corp., Armonk, NY, USA).

\section{RESULTS}

\section{Demographics}

Unilateral CAA combined with microtia were identified in 89 patients, 61 of whom were included in this study. The mean age at the time of diagnosis was $15.4 \pm 9.6$ years. Anomalies were present on the right side in 39 patients $(63.9 \%)$ and on the left side in the remaining patients. On the pathologic side, the mean Jahrsdoerfer score was $8.5 \pm 2.3$ and the mean Marx grade was $2.7 \pm 0.6$. The extent of inferior displacement of the mastoid tegmen on the pathologic side was $28.9 \% \pm 16.8 \%$, which was significantly greater $(P<0.05)$ than that on the unaffected side $(17.3 \% \pm 11.2 \%)$. The level of air conduction of PTA on the pathologic side was $69.6 \pm 13.1 \mathrm{~dB}$, which was significantly higher $(P<0.001)$ than that on the unaffected side $(11.1 \pm 10.1 \mathrm{~dB})$. The mean air-bone gap on the pathologic side was $58.4 \pm 10.7 \mathrm{~dB}$. The demographic data are summarized in Table 1.

\section{Inner ear anomalies}

Results from the analysis of the inner ear, facial nerve, and vascular anomalies with TBCT are presented in Table 2. Of the 61

Table 1. Demographic data for 61 patients with unilateral congenital aural atresia combined with microtia

\begin{tabular}{lc}
\hline Variable & Value \\
\hline Age (yr) & $15.4 \pm 9.6$ \\
Female:male & $39: 22$ \\
Anomaly side (right:left) & $39: 22$ \\
Jahrsdoerfer score & $8.5 \pm 2.3$ \\
Marx grade & $2.7 \pm 0.6$ \\
Extent of the inferior displacement of the mastoid tegmen (\%) \\
Pathologic side & $28.9 \pm 16.8^{*}$ \\
Unaffected side & $17.3 \pm 11.2$ \\
Pure tone audiometry (dB) & \\
AC, pathologic side & $69.6 \pm 13.1^{\star \star *}$ \\
BC, pathologic side & $10.6 \pm 11.7$ \\
AC, unaffected side & $11.1 \pm 10.1$ \\
BC, unaffected side & $7.1 \pm 10.9$ \\
ABG, pathologic side & $58.4 \pm 10.7$ \\
\hline
\end{tabular}

Values are presented as the mean \pm standard deviation.

$\mathrm{AC}$, air conduction; $\mathrm{BC}$, bone conduction; $\mathrm{ABG}$, air-bone gap.

The extent of the inferior displacement of the mastoid tegmen on the pathologic side was significantly higher than on the unaffected side $\left({ }^{*} P<0.05\right)$ and air conduction according to pure tone audiometry on the pathologic side was significantly higher than on the unaffected side $\left({ }^{* \star *} P<0.001\right)$. enrolled patients, 14 (23.0\%) had inner ear anomalies. Lateral semicircular canal (LSCC) dysplasia was observed in $10(16.4 \%)$ ears (Fig. 2). The incidence was significantly higher on the pathologic side than on the unaffected side $(P=0.002)$. No other semicircular canal (superior and posterior) dysplasias were observed. A dilated vestibule was identified on the pathologic side of four (6.6\%) ears (Fig. 3). Furthermore, a dilated vestibule was also incidentally found in one unaffected ear. The rate was not significantly different between the two sides $(P=0.321)$. A facial nerve anomaly was found in only one case $(1.6 \%)$, where medio-inferior displacement was observed in the tympanic segment of the facial nerve (Fig. 4). All vascular anomalies were identified in the HRJB, with a HRJB found in 15 ears (24.6\%) on the pathologic side (Fig. 5). The incidence rate on the pathologic side was significantly higher $(P<0.001)$ than that on the unaffected side.

\section{Clinical manifestations}

The results of the multiple logistic regression analysis of clinical manifestations associated with Jahrsdoerfer score are presented

Table 2. Results of analysis of inner ear anomalies in temporal bone computed tomography

\begin{tabular}{lccc}
\hline Anomaly & Pathologic side & $P$-value & Unaffected side \\
\hline Inner ear anomaly & $14(23.0)$ & - & 1 \\
SCC dysplasia & $10(16.4)$ & - & \\
Superior SCC & & & \\
Posterior SCC & & & \\
Lateral SCC & $10(16.4)$ & $0.002^{* *}$ & 1 \\
Vestibular dysplasia & $4(6.6)$ & 0.321 & \\
Facial nerve anomaly & $1(1.6)$ & & \\
HRJB & $15(24.6)$ & $<0.001^{* * *}$ & \\
\hline
\end{tabular}

Values are presented as number (\%).

SCC, semicircular canal; HRJB, high-riding jugular bulb.

The incidence of inner ear anomalies on the pathologic side was $23.0 \%$ in patients with unilateral congenital aural atresia combined with microtia. The incidence of lateral SCC dysplasia $\left(16.4 \%,{ }^{*} P=0.002\right)$ and HRJB $\left(24.6 \%\right.$, $\left.{ }^{* *} P<0.001\right)$ on the pathologic side was significantly higher than that on the unaffected side.
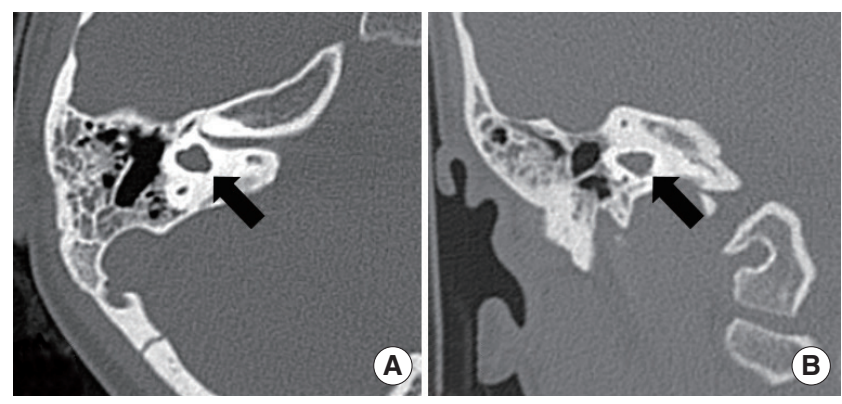

Fig. 2. High-resolution temporal bone computed tomography images of unilateral congenital aural atresia combined with microtia. Axial (A) and coronal (B) views showing the dysmorphic features of the lateral semicircular canal (arrows). 

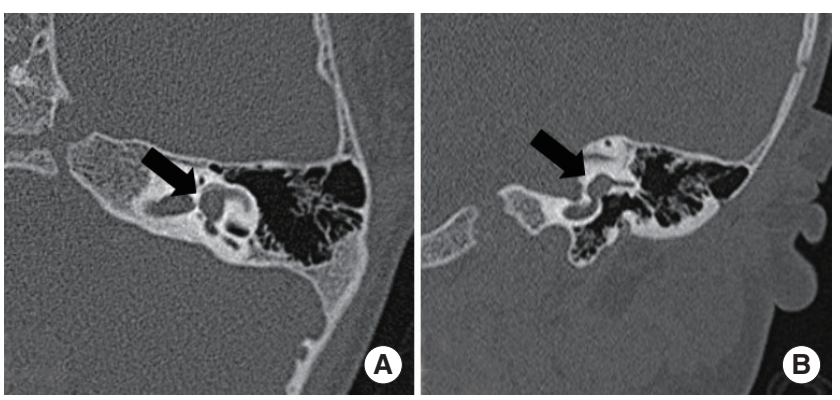

Fig. 3. High-resolution temporal bone computed tomography images of unilateral congenital aural atresia combined with microtia. Axial (A) and coronal (B) views showing the dilated vestibular morphology (arrows).
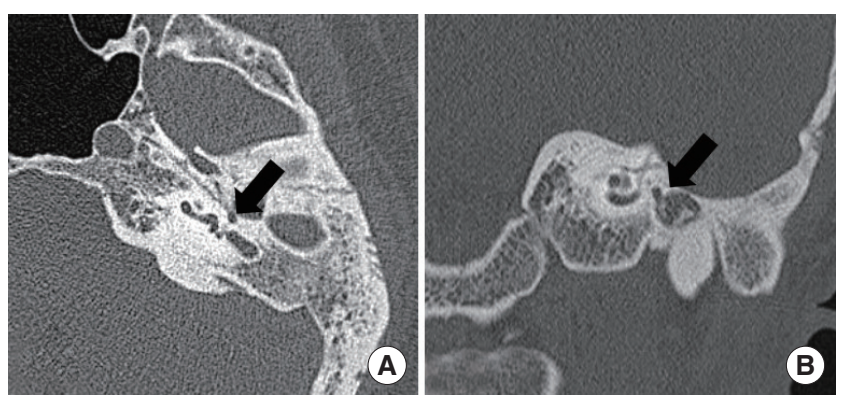

Fig. 4. High-resolution temporal bone computed tomography images of unilateral congenital aural atresia combined with microtia. Axial (A) and coronal (B) views showing the medio-inferiorly displaced tympanic segment of the facial nerve (arrows).

in Table 3. The presence of LSCC dysplasia was significantly associated with a lower Jahrsdoerfer score (odds ratio, $0.66 ; P=$ 0.004). However, the Jahrsdoerfer score was not significantly related to the presence of a HRJB. Furthermore, neither the Marx grade, nor the extent of inferior displacement of the mastoid tegmen was significantly correlated with LSCC dysplasia or the presence of a HRJB.

\section{DISCUSSION}

The current study revealed that the incidence of inner ear anomalies was significantly greater on the pathologic side than on the unaffected side. The incidence of LSCC dysplasia and HRJB was considerably higher on the pathologic side than on the unaffected side. LSCC dysplasia was significantly associated with a lower Jahrsdoerfer score.

The incidence of inner ear anomalies $(23.0 \%)$ determined by this study is similar to that reported in a previous study (prevalence rate, $22 \%$ in patients with CAA) [7]. The incidence of LSCC dysplasia (16.4\%) is also in close agreement with that found by an earlier study (14.8\%) [3]. However, these previous reports included patients with bilateral CAA. In a recent study,
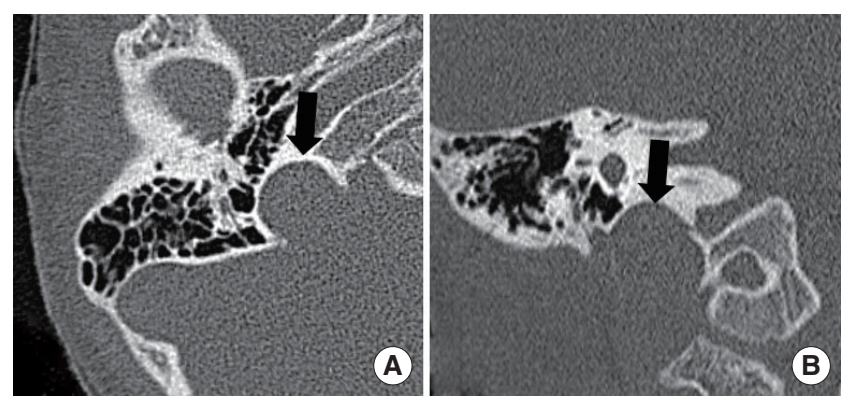

Fig. 5. High-resolution temporal bone computed tomography images of unilateral congenital aural atresia combined with microtia. Axial (A) and coronal (B) views showing high-riding jugular bulb (arrows).

Table 3. Results of multiple logistic regression analysis of clinical manifestations according to the Jahrsdoerfer score

\begin{tabular}{lcccc}
\hline Parameter & B & SE & Exp (B) & $P$-value \\
\hline LSCC dysplasia & -0.416 & 0.144 & 0.660 & $0.004^{\star \star}$ \\
HRJB & 0.128 & 0.167 & 1.136 & 0.444 \\
\hline
\end{tabular}

LSCC, lateral semicircular canal; HRJB, high-riding jugular bulb.

The presence of LSCC dysplasia was statistically significant in patients with lower Jahrsdoerfer score (odds ratio, 0.66; ${ }^{* *} P=0.004$ ).

Halle et al. [8] found anomalies of the inner ear in only two (3\%) of 70 patients with isolated nonsyndromic unilateral CAA. This result suggests that although the development of the external ear is not embryologically related to that of the inner ear, inner ear anomalies may be more commonly observed in patients with unilateral CAA combined with microtia compared to patients with only unilateral CAA.

We incidentally found an inner ear anomaly (a dilated vestibule) in one patient (1.6\%) in an unaffected "normal" ear. The patient had a normal hearing threshold in the unaffected ear and did not complain of any otologic symptoms, such as dizziness. In a previous study of cases of unilateral CAA or microtia, anomalies of the inner ear in the unaffected ear varied from $1.4 \%$ to $74 \%[8]$. These anomalies, typically asymptomatic, are not clinically meaningful. The authors found that the rate of inner ear anomalies was very low on the unaffected side in patients with unilateral CAA combined with microtia.

The Jahrsdoerfer criteria for TBCT are widely used for anatomic grading to determine the need for atresiaplasty in CAA. Recently, however, several authors have suggested that other parameters, such as the size of the IS joint angle and the extent of inferior displacement of the mastoid tegmen, are associated with the severity and hearing outcomes of CAA [12-14]. In addition, according to the increase of aMEI, a new grading system such as an aMEI score have been proposed and used.The aMEI score is more focused on the presence of coupling structures and mastoid aeration than the Jahrsdoerfer grading system [17].

Atresiaplasty and aMEI for CAA are challenging procedures 
for otologists. Because of unusual anatomical variation and dysmorphic features, iatrogenic injuries and severe complications can develop during surgery. This study showed a relatively high incidence of HRJB $(24.6 \%)$ on the pathologic side. Therefore, regarding safety in the operating field, surgeons must be aware of the possibility of vascular variation and massive bleeding from iatrogenic vessel injuries. In addition, facial nerve anomalies should always be considered. Facial nerve anomalies in patients with unilateral CAA combined with microtia are relatively low (1.6\% in this study), but according to previous studies, the incidence has ranged from $25 \%$ to $52 \%$ in CAA [18]. Furthermore, these patients have been reported to have more anterolateral displacement of the facial nerve [19]. Therefore, a comprehensive analysis of preoperative TBCT and careful inspection during surgery are crucial to avoid facial nerve damage.

This study has some limitations. The small patient population and the retrospective review, which shows that there was a lack of information on clinical characteristics related to the inner ear anomalies. However, the benefits included the use of a single TBCT protocol, a hearing test, and joint review of TBCT by an experienced otologist and radiologist. Additionally, limiting the study to patients with unilateral CAA combined with microtia was valuable. Further studies are needed to find the clinical characteristics of inner ear anomalies in patients with unilateral CAA combined with microtia.

In conclusion, this study showed that inner ear anomalies are relatively common in patients with unilateral CAA combined with microtia, with LSCC dysplasia being the most common anomaly. Vascular anomalies are also common and are present in HRJB. Furthermore, LSCC dysplasia is associated with a lower Jahrsdoerfer score, which may be the result of the developmental relationship between the middle ear and the inner ear.

\section{CONFLICT OF INTEREST}

No potential conflict of interest relevant to this article was reported.

\section{ACKNOWLEDGMENTS}

This research was supported by the Basic Science Research Program through the National Research Foundation of Korea (NRF) funded by Korea Evaluation Institute of Industrial Technology (R1621961), Korea Health Industry Development Institute (R1606511) and Korea University research grant (K1609821, Q1620561), Seoul, Republic of Korea.

The authors are grateful to Sung-Hye You (Department of Radiology, Korea University Anam Hospital).

\section{ORCID}

Euyhyun Park https://orcid.org/0000-0003-4373-6942

Gi Jung Im https://orcid.org/0000-0002-9457-4253

\section{REFERENCES}

1. Schuknecht HF. Congenital aural atresia. Laryngoscope. 1989 Sep; 99(9):908-17.

2. Genc S, Kahraman E, Ozel HE, Arslan IB, Demir A, Selcuk A. Microtia and congenital aural atresia. J Craniofac Surg. 2012 Nov;23(6): 1733-5.

3. Vrabec JT, Lin JW. Inner ear anomalies in congenital aural atresia. Otol Neurotol. 2010 Dec;31(9):1421-6.

4. MayerTE, Brueckmann H, Siegert R, Witt A, Weerda H. High-resolution CT of the temporal bone in dysplasia of the auricle and external auditory canal. AJNR Am J Neuroradiol. 1997 Jan;18(1):53-65.

5. Yuen HY, Ahuja AT, Wong KT, Yue V, van Hasselt AC. Computed tomography of common congenital lesions of the temporal bone. Clin Radiol. 2003 Sep;58(9):687-93.

6. Naunton RF, Valvassori GE. Inner ear anomalies: their association with atresia. Laryngoscope. 1968 Jun;78(6):1041-9.

7. Phelps PD. Congenital lesions of the inner ear, demonstrated by tomography. Arch Otolaryngol. 1974 Jul;100(1):11-8.

8. Halle TR, Soares BP, Todd NW. Inner ear anomalies in children with isolated unilateral congenital aural atresia. Int J Pediatr Otorhinolaryngol. 2017 Apr;95:5-8.

9. Balaker AE, Roberson JB Jr, Goldsztein H. Fibrous incudostapedial joint in congenital aural atresia. Otolaryngol Head Neck Surg. 2014 Apr;150(4):673-6.

10. Yellon RF, Branstetter BF 4th. Prospective blinded study of computed tomography in congenital aural atresia. Int J Pediatr Otorhinolaryngol. 2010 Nov;74(11):1286-91.

11. Jahrsdoerfer RA, Yeakley JW, Aguilar EA, Cole RR, Gray LC. Grading system for the selection of patients with congenital aural atresia. Am J Otol. 1992 Jan;13(1):6-12.

12. Dedhia K, Yellon RF, Branstetter BF, Egloff AM. Anatomic variants on computed tomography in congenital aural atresia. Otolaryngol Head Neck Surg. 2012 Aug;147(2):323-8.

13. Kim DW, Lee JH, Song JJ, Chang H, Choi YS, Jang JH, et al. Continuity of the incudostapedial joint: a novel prognostic factor in postoperative hearing outcomes in congenital aural atresia. Acta Otolaryngol. 2011 Jul;131(7):701-7.

14. Ju YH, Park E, Park S, Jung K, Lee K, Im GJ. The extent of inferior displacement of the mastoid tegmen is related to the severity of congenital aural atresia. Acta Otolaryngol. 2014 Mar;134(3):244-9.

15. Sennaroglu L, Saatci I. A new classification for cochleovestibular malformations. Laryngoscope. 2002 Dec;112(12):2230-41.

16. Brook CD, Buch K, Kaufmann M, Sakai O, Devaiah AK. The prevalence of high-riding jugular bulb in patients with suspected endolymphatic hydrops. J Neurol Surg B Skull Base. 2015 Dec;76(6):471-4.

17. Frenzel H, Sprinzl G, Widmann G, Petersen D, Wollenberg B, Mohr C. Grading system for the selection of patients with congenital aural atresia for active middle ear implants. Neuroradiology. $2013 \mathrm{Jul}$; 55(7):895-911.

18. Yu Z, Han D, Gong S, Wang Z, Dai H, Zhao S, et al. Facial nerve course in congenital aural atresia: identified by preoperative CT scanning and surgical findings. Acta Otolaryngol. 2008;128(12):1375-80.

19. Takegoshi H, Kaga K, Kikuchi S, Ito K. Facial canal anatomy in patients with microtia: evaluation of the temporal bones with thin-section CT. Radiology. 2002 Dec;225(3):852-8. 